\title{
Búsqueda de empleo, capital social y salarios en México
}

Recibido: 19 de marzo de 2021 - Aceptado: 28 de abril de 2021

Doi: https://doi.org/10.12804/revistas.urosario.edu.co/economia/a.10470

\author{
Humberto Charles-Leija* \\ Aldo Josafat Torres García ${ }^{\dagger}$ \\ David Castro-Lugo $\ddagger$
}

Resumen

La investigación tiene como objetivo verificar la existencia de disparidades salariales en México a partir del canal de búsqueda de empleo. En este caso, el análisis se centró en las redes de contactos de los individuos, emplea datos de la Encuesta Nacional de Ocupación y Empleo (ENOE) del 2019, estimando una ecuación de determinación salarial semilogarítmica. Los resultados exhiben una prima salarial por haber obtenido empleo mediante Internet $(12.4 \%)$, un sindicato $(6.7 \%)$, bolsa privada ( $8.9 \%)$ y contactos personales $(6.7 \%)$. Cuando se distingue por edad, se observa que las personas con más de 30 años reportan el mayor impacto positivo de encontrar empleo a través de sus redes de contactos.

Palabras clave: búsqueda de empleo; salario; capital social; redes de contactos.

Clasificación JEL: E24, Z13, J24.

* Instituto de Ciencias del Bienestar y la Felicidad, Universidad Tecmilenio y Escuela de Negocios, Tecnológico de Monterrey. Dirección: Tecmilenio "Las Torres", edificio propósito, $4 .^{\circ}$ piso, Paseo del Acueducto 2610, Del Paseo Residencial, 64909. Monterrey, México. Teléfono: +52 8110520000 Email: humbertocharles@yahoo.com orcid: https://orcid.org/0000-0003-1730-8661

† Facultad de Economía Internacional, Universidad Autónoma de Chihuahua. ORCID: https://orcid.org/0000-0002-2936-7485

$\ddagger$ Centro de Investigaciones Socio-económicas, Universidad Autónoma de Coahuila. ORCID: https://orcid.org/0000-0003-0130-7197

Para citar este artículo: Charles-Leija, H., Torres, A., \& Castro, D. (2021). Búsqueda de empleo, capital social y salarios en México. Revista de Economía del Rosario, 24(2), 1-24. https://doi.org/10.12804/revistas.urosario.edu.co/economia/a.10470 


\title{
Job Search Methods, Social Capital and Wages in Mexico
}

\begin{abstract}
The purpose of this study is to verify the existence of wage disparities from the job search channel and to focus on the social networks of individuals. The data used comes from the 2019 National Survey of Occupation and Employment (ENOE), and a semi-logarithmic wage determination equation was estimated. The results show a premium salary according to the channel the employment was obtained: Internet $(12.4 \%)$, a union $(6.7 \%)$, private stock market $(8.9 \%)$, and personal contacts $(6.7 \%)$. When we distinguished by age, those over 30 report the greatest positive impact of finding employment through their networks.
\end{abstract}

Keywords: job search; wage; social capital; networking.

JEL classification: E24, Z13, J24.

\section{Busca de emprego, capital social e salários no México}

\section{Resumo}

A investigação tem como objetivo verificar a existência de disparidades salariais no canal de busca de emprego. A análise está focada nas redes sociais dos indivíduos. Os dados empregados pertencem à Pesquisa Nacional de Ocupação e Emprego (ENOE), de 2019, e uma equação semilogarítmica de determinação de salários é estimada. Os resultados mostram um bônus salarial por ter obtido emprego pela Internet (12.4\%), sindicato (6.7\%), bolsa privada ( $8.9 \%$ ) e contatos pessoais (6.7\%). Quando diferenciados por idade, observa-se que os maiores de 30 anos relatam o maior impacto positivo de conseguir um emprego por meio de suas networking.

Palavras-chave: busca de trabalho; salário; capital social; networking.

Classificação JEL: E24, Z13, J24. 


\section{Introducción}

Los individuos buscan el empleo con mejor salario. Entre los factores fundamentales que se han propuesto para explicar el ingreso laboral de las personas, están la escolaridad y la experiencia (Mincer, 1974). Asimismo, se han abordado otros elementos, tales como tamaño de la empresa, pertenencia a sindicatos, duración de la jornada laboral, localidad, sector de actividad, estado civil, sexo, competencias tecnológicas, e incluso, la dualidad del mercado laboral (formal/informal) (Bracho \& Zamudio, 1994; Barceinas Paredes, 2002; Villareal, 2010; Huesca \& Ochoa, 2016; Torres \& Félix, 2017).

En este sentido, un elemento que ha cobrado relevancia entre los analistas laborales es el de capital social al interior del mercado de trabajo; tema que, para el caso de México ha sido escasamente explorado. Dicho lo anterior, el presente trabajo indaga en las diferencias salariales a partir de los métodos de búsqueda de empleo, haciendo especial hincapié en las referencias dadas por amigos o familiares al interior de las unidades económicas. En específico, este documento pretende responder las siguientes preguntas: ¿Existe una prima salarial por obtener empleo a través de los contactos personales?, y de ser así, ¿cuál es su magnitud?

La literatura internacional acerca de capital social y mercado laboral, pone especial atención a la asociación entre amigos y familiares como referentes de ofertas de trabajo. Por lo tanto, en México es pertinente profundizar en los mecanismos de búsqueda de trabajo y en el papel que desempeña el capital social (Charles-Leija et al., 2018; Méndez et al., 2018; Rodríguez-Villalobos \& Rangel-González, 2020).

Dicho lo anterior, el objetivo de la investigación es identificar si existe un impacto salarial de acuerdo con el método utilizado para encontrar trabajo. Para esto, el estudio emplea los microdatos de la ENOE 2019 primer trimestre (INEGI, 2019). Derivado del análisis, se encontró una prima salarial por haber obtenido empleo a partir de la referencia de un amigo o familiar de un $6.7 \%$ en la estimación más robusta. Es importante destacar que la mayor contribución a partir del método de búsqueda la ofrece Internet, con un $12.4 \%$, al igual que el servicio de empleo temporal del gobierno (12.4\%), le sigue la bolsa privada ( $8.91 \%$ ) y los medios de comunicación $(8 \%)$.

Un hallazgo interesante, se observó en la diferencia del método más utilizado para buscar empleo por los desempleados, esto es, acudir directamente al lugar de trabajo (con un $65 \%$ de referencias) y el método por el cual los trabajadores ocupados encontraron empleo, por aviso de un amigo o familiar (55\% de menciones). De esta manera se aprecia que modificando los 
mecanismos de búsqueda laboral se pueden obtener mejores resultados, tanto en términos del tiempo de búsqueda de empleo, como en las remuneraciones.

El trabajo se divide en cinco secciones: la primera es introductoria; la segunda hace un breve repaso de la literatura sobre el tema; la tercera describe la base de datos, hipótesis y metodología utilizada; la cuarta presenta y discute los resultados, y por último, algunas conclusiones de la investigación.

\section{Revisión de la literatura}

Al respecto de los medios para encontrar empleo y la importancia de las redes de contactos, existe una literatura amplia (Addison \& Portugal, 2002; Aguilera, 2002; Bachmann \& Baumgarten, 2012; Calvó-Armegol \& Jackson, 2004). Una distinción clave al respecto la realizó Rees (1966), al diferenciar los canales de búsqueda de información en formales e informales. La propuesta señala que los medios formales comprenden los servicios estatales de empleo, agencias de colocación, avisos en los periódicos, reuniones sindicales y, departamentos de vinculación escolar; mientras que, los medios informales hacen alusión a referencias de empleados y presentarse directamente en el establecimiento.

En lo que refiere al presente estudio se entenderán como medios formales aquellos que contienen información pública de las firmas, Internet y los servicios de empleo temporal. En cuanto a los medios informales, se considerará cuando el desempleado recibe una oferta laboral de parte del empleador, ya que ello representa que un miembro de la firma contratante tiene información sobre las competencias y talentos del buscador de empleo. Ahora bien, los desempleados recurren a alguno o varios de los mecanismos comentados previamente. La literatura sugiere que uno de los más populares es el de las redes de contactos, debido a su bajo costo y libre acceso (Méndez et al., 2018). A continuación, se detallan un poco más los canales de búsqueda de empleo.

\subsection{Métodos de búsqueda de empleo}

\subsubsection{Acudir al lugar}

Como se mencionó existen diversas maneras de buscar empleo en la actualidad. Una de las más tradicionales es dirigirse directamente al lugar a ofertar la propia fuerza de trabajo. Los individuos que recurren a este canal consumen tiempo y dinero para transportarse a los distintos establecimientos en los que les interesa trabajar. Ello no solo repercute en los gastos en los que incurre el buscador de trabajo, sino que también puede implicar un elevado 
nivel de desconocimiento sobre las condiciones de la empresa a la que se acerca. La misma situación ocurre para la firma.

\subsubsection{Agencia o bolsa de trabajo}

Las bolsas de trabajo representan una red formal que sirve de vínculo entre desempleados y empleadores. Una particularidad que manifiesta este canal de búsqueda es que corresponde con empresas que tienen como misión ser el puente entre individuos y firmas. Dado lo anterior, el éxito de la agencia depende de la calidad de los emparejamientos que realiza.

\subsubsection{Servicio público}

Existen actividades en la economía que son suministradas tanto por el Estado como por agentes privados. En términos de enlaces laborales, los organismos gubernamentales ofrecen servicios públicos de empleo. Sin embargo, los incentivos entre las oficinas de empleo estatales y las privadas pueden diferir, ya que las primeras dependen de un aparato burocrático y las segundas de la eficiencia al realizar su tarea. La literatura ha mostrado que las agencias públicas de colocación pueden ser menos eficientes que las provenientes de la iniciativa privada (Addison \& Portugal, 2002).

\subsubsection{Empleo temporal}

Otra opción propuesta por entidades gubernamentales es el Programa de Empleo Temporal (PET), enfocado en "mitigar el impacto económico y social de las personas de 16 años de edad o más que vean disminuidos sus ingresos o su patrimonio ocasionado por situaciones sociales y económicas adversas, emergencias o desastres" (Empleo, 2020).

\subsubsection{Sindicato}

Un factor que puede contribuir a que las personas obtengan mayores beneficios, parte de que algunas instituciones tienen políticas al respecto de heredar plazas a familiares de trabajadores. Estudios previos han apuntado que pertenecer a un sindicato genera en la mayoría de los individuos premios salariales, provocando diferencias significativas en el ingreso laboral entre quién está y quién no está vinculado a un gremio sindical (Burgos \& Mungaray, 2007; Esquinca \& Valdivia, 2006). 


\subsubsection{Internet}

El uso de Internet como canal de búsqueda requiere cierto grado de instrucción previa. Esta herramienta implica varios costos, el primero relacionado con el aprendizaje para emplear el medio, tal costo puede ser insignificante para los individuos que manipulan los servicios de la web con frecuencia, sin embargo, en México aún existe una brecha digital que le impide a algunos sectores de la población familiarizarse con ciertas tecnologías de la información (Torres \& Félix, 2017). Otros costos van de la mano con el acceso. En Estados Unidos, se documentó que a finales de la década de 1990 era ineficiente buscar empleo mediante Internet, no obstante, ese medio de búsqueda resultó mejorar su eficiencia para finales de la primera década del siglo XxI (Kuhn \& Mansour, 2014).

\subsubsection{Periódico, radio, anuncio}

Del lado de las empresas hay distintas maneras de informar sobre sus vacantes. Un canal formal que aún es ampliamente utilizado por las firmas, es la difusión por medios electrónicos e impresos, tales como radio, televisión y periódicos. A este respecto los datos mostraron que más del $9 \%$ de los empleados con nivel de escolaridad de secundaria y superior, obtuvieron su empleo a través de este medio.

\subsection{Los canales informales como medio eficiente de emparejamiento}

Las firmas desconocen el nivel de habilidades y compromiso que poseen los individuos. Por ello requieren de elementos que faciliten dicha información. Algunas empresas recurren a cartas de recomendación, y en ocasiones, otro esquema que puede funcionar es escuchar las sugerencias hechas por empleados que han probado capacidades, competencias y elevados niveles de compromiso (Goyal, 2007).

Los individuos empleados compartirán con sus conocidos desempleados información sobre vacantes, a menos que ellos mismos puedan aprovechar la oportunidad (Calvó-Armegol \& Jackson, 2004). Mientras tanto, para los desempleados es crucial tener una red de contactos que le informe al respecto del mercado laboral, es decir, sobre datos que no estén disponibles en medios públicos. Entre mayor es la red, más alta es la probabilidad de recibir una oferta de trabajo (Fontaine, 2008).

La intención de los individuos al integrarse al mercado laboral normalmente es obtener la mayor remuneración posible. Sin embargo, el salario de 
los empleados está influido por su nivel de calificación, así como la coyuntura que atraviese la región. En tal sentido es relevante el capital humano que posea cada trabajador, en términos de educación, experiencia, salud, competencias, habilidades y talentos personales. Aunado a esto, Montgomery (1991) sugirió que el salario, además de estar determinado por la habilidad y capacidades productivas, presenta un sesgo originado por las relaciones sociales, en la cual los varones presentan ventaja.

$\mathrm{Al}$ respecto, se ha identificado que las redes de amistad están relacionadas positivamente con la participación en la fuerza de trabajo (Aguilera, 2002) y que el capital social tiene un efecto directo en el salario (Aguilera \& Massey, 2003). Así mismo, es de resaltar que en este trabajo se entenderá el capital social como las personas que pertenecen a la red de amigos, familiares y conocidos de un individuo que le pueden compartir información sobre el mercado laboral.

\subsection{Revisión de antecedentes empíricos}

Los estudios empíricos apuntan a que cerca del $50 \%$ de los trabajadores encontraron por medio de sus amigos, familiares o conocidos (Loury, 2006; Montgomery, 1991; Rees, 1966). Además, se ha identificado que quienes obtienen empleo por ese canal regularmente alcanzan mayor antigüedad en las firmas (Loury, 2006). Algunas investigaciones anteriores han revelado que obtener empleo por medio de familiares y amigos puede representar una prima salarial. Bowlus y Ontario (1997) encontraron que los métodos de búsqueda de empleo pueden tener un impacto salarial cercano al $25 \%$ entre estadounidenses con nivel educativo de preparatoria y licenciatura.

Adicional a la información sobre el mercado laboral que generan las redes familiares y sociales, se ha visto que las personas que ingresan a trabajar con individuos que ya conocían son más productivas, lo cual, puede deberse a que el ambiente laboral fortalecido por redes de contactos previas genera amenidades no pecuniarias que incentivan a los trabajadores, dicha situación tiene mayor relevancia en personas de baja calificación (Hellerstein et al., 2013). De igual manera, los individuos sienten más satisfacción de trabajar en una empresa donde tienen amigos, incluso les lleva a tener un mayor nivel de compromiso con la firma (Rath \& Harter, 2010).

Otros estudios han identificado que, entre alumnos de habilidades similares, aquellos que asistieron a universidades más selectivas obtuvieron mayores ingresos en sus empleos posteriores (Dale \& Krueger, 2002), y que existe una correlación entre pertenecer a una fraternidad y obtener un primer empleo mejor remunerado (Marmaros \& Sacerdote, 2002). 
En Europa, se han identificado salarios más altos para las personas que obtuvieron su empleo a través de la recomendación, la diferencia es mayor para Austria, Bélgica y Holanda. El efecto sobre el salario es más elevado en países con más intermediarios dentro del mercado laboral (Pellizzari, 2010). También, se ha señalado que las características observables de los trabajadores determinan apenas un tercio de su salario, el resto podría explicarse por la heterogeneidad no observada de los trabajadores, heterogeneidad de las firmas y, efectos debidos a fricciones de búsqueda (Fontaine, 2008).

Algunos estudios han identificado que inmediatamente después del bachillerato los lazos de amistad no generan beneficios pecuniarios, pero con el tiempo se generan impactos salariales (Rosenbaum et al., 1999). Por su parte, Michael Aguilera y Massey (2003) identificaron que los lazos de familia y amigos en los Estados Unidos tuvieron un efecto positivo y significativo sobre las tasas salariales de los inmigrantes mexicanos, lo cual representó un incremento en el ingreso de aproximadamente el $23 \%$ para los indocumentados.

De manera contraria, Addison y Portugal (2002) identificaron un efecto negativo en el salario de los trabajadores portugueses, cuando el empleo había sido obtenido por medio de los siguientes mecanismos: (i) redes de amistad, (ii) agencias públicas de empleo, y (iii) directamente el establecimiento. Por otra parte, Bridges y Villemez (1986) en un estudio realizado para el área metropolitana de Chicago, encontraron que los lazos de amistad no son un mediador relevante entre el capital humano y el ingreso obtenido, es decir, que el obtener trabajo mediante amigos o familiares, no representa una prima salarial para los individuos.

\section{Datos y metodología}

En la parte empírica de este documento, se utilizaron los microdatos de la ENOE para el primer trimestre del 2019. La encuesta tiene representatividad en el ámbito nacional y considera a los individuos de 12 años en adelante. Esta encuesta proporciona la información referente al salario, medios de búsqueda y obtención de empleo, así como un conjunto de características sociodemográficas necesarias para el análisis descriptivo y las estimaciones posteriores.

La tabla 1 representa los porcentajes y salarios promedio de los individuos a partir del medio utilizado para obtener su empleo actual. En la tabla 1 se observa que, el $54.76 \%$ de los ocupados, encontró su trabajo por medio de un familiar, amigo o conocido. Mientras que el $19.56 \%$ lo obtuvo acudiendo directamente al lugar de trabajo. Por su parte, al $11.64 \%$ le ofrecieron el empleo. 
De forma tal que, cerca del $86 \%$ de los individuos empleados consiguieron su trabajo por alguno de estos tres canales de búsqueda de empleo informal y, más de la mitad lo obtuvo a través de contactos personales. ${ }^{1}$

Tabla 1. Medio por el cual encontró el empleo actual, México 2019

\begin{tabular}{lcc}
\hline \multicolumn{1}{c}{ Opción de búsqueda } & Porcentaje & Salario promedio \\
\hline Acudir al lugar & 19.56 & $5.638,00$ \\
Agencia o bolsa de trabajo & 0.4 & $5.947,20$ \\
Servicio público de colocación & 0.16 & $5.561,57$ \\
Empleo temporal & 0.22 & $4.908,94$ \\
Sindicato o gremio & 1.09 & $5.625,08$ \\
Internet & 2.3 & $6.606,35$ \\
Periódico, radio, anuncio & 9.25 & $5.826,67$ \\
Familiar, amigo o conocido & 54.76 & $5.614,01$ \\
Empleo ofrecido & 11.64 & $5.275,75$ \\
Otro medio & 0.62 & $5.500,94$ \\
\hline
\end{tabular}

Fuente: elaboración propia con datos de ENOE (INEGI, 2019).

Una pregunta que puede surgir al evaluar los esquemas más eficientes para encontrar empleo es: ¿El canal de búsqueda cambia por nivel educativo? La tabla 2 confirma lo propuesto por Rees (1966), en el sentido de que alrededor de la mitad de la población encuentra empleo por medio de sus contactos personales y que ello ocurre en todos los niveles educativos.

La tabla 2 describe los métodos de búsqueda utilizados a partir del nivel de instrucción de los trabajadores. Es posible ver que las personas de mayor nivel educativo accedieron a los salarios promedio más altos mediante servicios públicos de colocación e Internet, mientras que los menos escolarizados obtienen los mayores ingresos promedio a través de bolsa de trabajo o programas de empleo temporal del Gobierno. La opción de empleo temporal aparece como la alternativa menos recurrida, en prácticamente todos los niveles de instrucción, en tanto, la de menores ingresos es el servicio público de colocación para los ocupados de baja escolaridad y, bolsa de trabajo para media superior y superior. Cabe señalar que, en todos los niveles de

1 Para el análisis se empleó la pregunta "¿Cómo se enteró de este empleo?", del cuestionario ampliado. El $2.01 \%$ de los encuestados no sabía o no contestó el medio por el cual consiguió el empleo. 


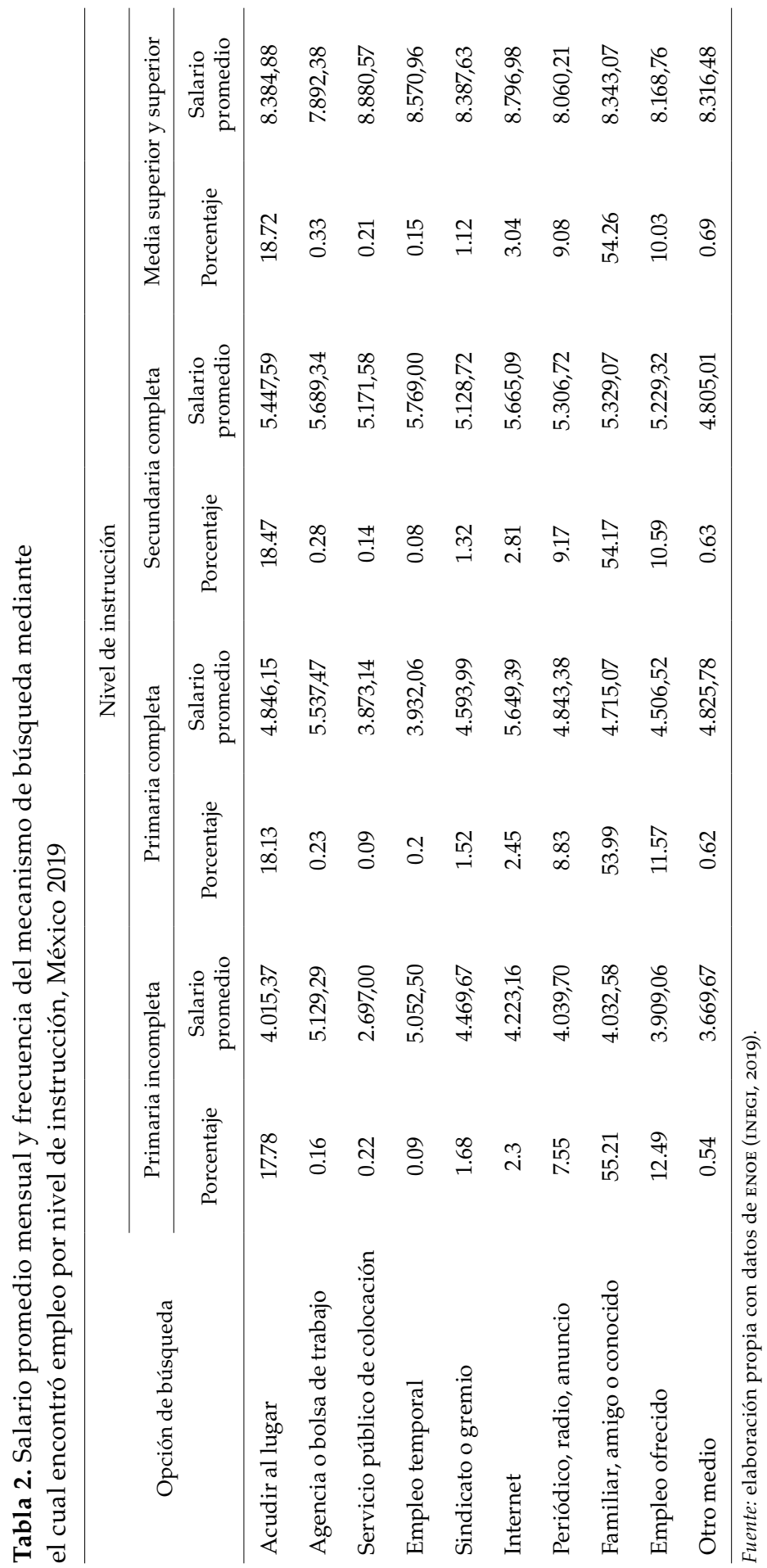


instrucción, más de la mitad de los trabajadores consiguió su empleo por medio de un familiar, amigo o conocido. ${ }^{2}$ Estos resultados apuntan a que la bolsa de trabajo e Internet, permiten salarios elevados tanto para los no calificados como para los calificados, con la excepción del servicio público de colocación para los de mayor instrucción.

De igual forma, la tabla 2 sugiere que el canal que más permite salir de las filas del desempleo es el de contactos personales, incluso, entre individuos de alta calificación. Así mismo, conforme se incrementa el nivel educativo de las personas, el porcentaje de trabajadores a quienes les fue ofrecido el empleo se reduce, caso contrario a lo que sucede con Internet, el cual se muestra creciente con la escolaridad. Por su parte, acudir al lugar de trabajo sigue siendo una opción de búsqueda muy recurrente, puesto que, alrededor del $18 \%$ de los trabajadores consiguieron así su empleo, independientemente del nivel escolar. En suma, más del $72 \%$ de los ocupados por nivel de instrucción encontró su empleo vía contactos personales y visitando directamente el lugar de trabajo.

\subsection{Metodología}

Para esta investigación se utilizó un modelo de determinación salarial tipo Mincer (1974), basado en la función de ingresos del capital humano como se muestra a continuación, en la cual, se incorporó el mecanismo de búsqueda de empleo como variable independiente:

$$
\begin{gathered}
\ln w=f\left(\text { escolaridad, experiencia, experiencia }{ }^{2}\right. \\
\text { medio_búsqueda, controles })
\end{gathered}
$$

De esta forma, la ecuación de estimación queda como sigue:

$$
y=\beta_{0}+\beta_{1} S+\beta_{2} E+\beta_{3} E^{2}+\delta_{1} m \_b u s q+\beta_{4} X+u
$$

Donde $y$ es el logaritmo natural del salario por hora trabajada, $S$ es la escolaridad de los individuos, $E$ representa la experiencia potencial del trabajador, ${ }^{3}$ la variable dicotómica $m \_b u s q$ representa el método de búsqueda

2 La tabla excluye a los individuos que no saben o no contestaron cómo se enteraron de su empleo.

3 La experiencia potencial es calculada mediante la fórmula $E=T-S-6$. Donde $T$ es la edad del individuo. $E^{2}$ representa los rendimientos decrecientes asociados a la 
a través del cual el trabajador consiguió su actual empleo: acudir directamente, mediante una bolsa privada, servicio público de colocación, sindicato, Internet, medios de comunicación, redes de contactos, ofrecimiento por parte de la empresa y otro. Por su parte, $X$ es un vector de variables referentes a características sociodemográficas de los individuos que inciden en la determinación salarial. Empíricamente, el modelo desagrega $S$ en niveles de instrucción, esto es, $S=S_{1}, S_{2}, S_{3}, S_{4}$, siendo en ese orden: primaria incompleta, primaria completa, secundaria completa, y media superior y superior. ${ }^{4}$ Por su parte, $u$ es la perturbación aleatoria con media cero y varianza constante. Respecto a los controles econométricos en cada estimación, se incorporan variables dummy de ocupación y de región; en el primer caso con base en el Sistema Nacional de Clasificación de Ocupaciones (sINCo), ${ }^{5}$ y en el segundo, considerando la regionalización de Hanson (2003).

\section{Resultados y discusión}

\subsection{Resultados}

Para contrastar la hipótesis se verificaron los microdatos provenientes de la ENOE 2019, con el objetivo de dimensionar el impacto del esquema de búsqueda sobre los salarios. Para ello, se considera la ecuación semilogarítmica planteada (sección 2), en la cual, se incorporan como regresores los canales de búsqueda de empleo y se desagrega la escolaridad por niveles de instrucción. ${ }^{6}$

Los resultados de la ecuación estimada por Mínimos Cuadrados Ordinarios (мсо) se presentan en la tabla 3 y los de la estimación ajustada se muestran en la tabla 4.

experiencia potencial. Esta variable es medida de esta manera, ya que la encuesta de ocupación y empleo en México, no capta de forma directa la experiencia laboral.

4 Un primer enfoque de la escolaridad por cohortes en la variable educación, es utilizado para México por Bracho y Zamudio (1994). Los niveles de instrucción son tomados directamente de ENOE.

5 https://www.snieg.mx/DocumentacionPortal/Normatividad/vigente/ sinco_2019.pdf

6 Sin embargo, cabe mencionar que existen variables no observables que pueden incidir en la explicación de la relación aquí planteada, relacionadas con "mejores habilidades sociales", y que los datos de la encuesta utilizada no captan. 
Tabla 3. Ecuación salarial por mсо considerando los mecanismos de búsqueda de empleo sobre el logaritmo natural del salario como variable dependiente

\begin{tabular}{|c|c|c|c|}
\hline & $\begin{array}{l}\text { (1) Mecanismo de } \\
\text { búsqueda }\end{array}$ & $\begin{array}{l}\text { (2) Mecanismo } \\
\text { de búsqueda, } \\
\text { ocupaciones }\end{array}$ & $\begin{array}{l}\text { (3) Mecanismo de } \\
\text { búsqueda, ocupa- } \\
\text { ciones y regiones }\end{array}$ \\
\hline \multirow{2}{*}{ Años de escolaridad } & 0.0690 & 0.0440 & 0.0430 \\
\hline & $(0.0001)$ & $(0.0001)$ & $(0.0001)$ \\
\hline \multirow{2}{*}{ Sexo } & 0.2080 & 0.2280 & 0.2230 \\
\hline & $(0.0004)$ & $(0.0004)$ & $(0.0004)$ \\
\hline \multirow{2}{*}{ Estado civil } & 0.0460 & 0.0530 & 0.0600 \\
\hline & $(0.0004)$ & $(0.0004)$ & $(0.0004)$ \\
\hline \multirow{2}{*}{ Experiencia } & 0.0170 & 0.0160 & 0.0160 \\
\hline & $(0.0000)$ & $(0.0000)$ & $(0.0000)$ \\
\hline \multirow{2}{*}{ Experiencia al cuadrado } & 0.0000 & 0.0000 & 0.0000 \\
\hline & $(0.0000)$ & $(0.0000)$ & $(0.0000)$ \\
\hline \multirow{2}{*}{ Empleo formal } & 0.3310 & 0.2580 & 0.2190 \\
\hline & $(0.0004)$ & $(0.0004)$ & $(0.0004)$ \\
\hline \multirow{2}{*}{ Sector agro } & -0.0580 & -0.0140 & -0.0110 \\
\hline & $(0.0001)$ & $(0.0002)$ & $(0.0002)$ \\
\hline \multirow{2}{*}{ Tamaño de empresa } & 0.0150 & 0.0080 & 0.0070 \\
\hline & $(0.0001)$ & $(0.0001)$ & $(0.0001)$ \\
\hline \multirow{2}{*}{ Logaritmo de horas trabajadas } & -0.5060 & -0.4900 & -0.4830 \\
\hline & $(0.0003)$ & $(0.0003)$ & $(0.0003)$ \\
\hline \multirow{2}{*}{ Ir directo al lugar } & 0.0930 & 0.0750 & 0.0700 \\
\hline & $(0.0039)$ & $(0.0037)$ & $(0.0036)$ \\
\hline \multirow{2}{*}{ bolsa_privada } & 0.1360 & 0.1150 & 0.0890 \\
\hline & $(0.0051)$ & $(0.0049)$ & $(0.0048)$ \\
\hline \multirow{2}{*}{ Servicios_publicos_colocación } & omitted & omitted & omitted \\
\hline & - & - & - \\
\hline \multirow{2}{*}{ empleo_temporal_gob } & 0.1520 & 0.1410 & 0.1240 \\
\hline & $(0.0060)$ & $(0.0058)$ & $(0.0057)$ \\
\hline \multirow{2}{*}{ Sindicato } & 0.0840 & 0.0760 & 0.0670 \\
\hline & $(0.0041)$ & $(0.0039)$ & $(0.0038)$ \\
\hline
\end{tabular}




\begin{tabular}{|c|c|c|c|}
\hline & $\begin{array}{l}\text { (1) Mecanismo de } \\
\text { búsqueda }\end{array}$ & $\begin{array}{l}\text { (2) Mecanismo } \\
\text { de búsqueda, } \\
\text { ocupaciones }\end{array}$ & $\begin{array}{l}\text { (3) Mecanismo de } \\
\text { búsqueda, ocupa- } \\
\text { ciones y regiones }\end{array}$ \\
\hline \multirow{2}{*}{ Internet } & 0.1620 & 0.1390 & 0.1240 \\
\hline & $(0.0040)$ & $(0.0038)$ & $(0.0038)$ \\
\hline \multirow{2}{*}{ medio_comunicion } & 0.1050 & 0.0890 & 0.0800 \\
\hline & $(0.0039)$ & $(0.0037)$ & $(0.0037)$ \\
\hline \multirow{2}{*}{ Familiar_o_amigo } & 0.0910 & 0.0750 & 0.0670 \\
\hline & $(0.0038)$ & $(0.0037)$ & $(0.0036)$ \\
\hline \multirow{2}{*}{ Empleo fue ofrecido } & 0.0570 & 0.0480 & 0.0470 \\
\hline & $(0.0039)$ & $(0.0037)$ & $(0.0037)$ \\
\hline \multirow{2}{*}{ otro } & 0.0830 & 0.0770 & 0.0750 \\
\hline & $(0.0044)$ & $(0.0042)$ & $(0.0042)$ \\
\hline \multirow{2}{*}{ d_ocup1 } & & -0.376 & -0.372 \\
\hline & & $(0.001)$ & $(0.001)$ \\
\hline \multirow{2}{*}{ d_ocup2 } & & -0.296 & -0.263 \\
\hline & & $(0.002)$ & (0.002) \\
\hline \multirow{2}{*}{ d_ocup3 } & & 0.000 & 0.000 \\
\hline & & (omitted) & (omitted) \\
\hline \multirow{2}{*}{ d_ocup4 } & & -0.567 & -0.566 \\
\hline & & $(0.001)$ & $(0.001)$ \\
\hline \multirow{2}{*}{ d_ocup5 } & & -0.706 & -0.719 \\
\hline & & $(0.001)$ & $(0.001)$ \\
\hline \multirow{2}{*}{ d_ocup6 } & & -0.851 & -0.853 \\
\hline & & $(0.001)$ & $(0.001)$ \\
\hline \multirow{2}{*}{ d_ocup7 } & & -0.660 & -0.670 \\
\hline & & $(0.002)$ & $(0.001)$ \\
\hline \multirow{2}{*}{ d_ocup8 } & & -0.757 & -0.769 \\
\hline & & $(0.001)$ & $(0.001)$ \\
\hline \multirow{2}{*}{ d_ocup9 } & & -0.672 & -0.663 \\
\hline & & $(0.002)$ & $(0.002)$ \\
\hline \multirow{2}{*}{ d_r1 } & & & 0.167 \\
\hline & & & $(0.001)$ \\
\hline
\end{tabular}




\begin{tabular}{|c|c|c|c|}
\hline & $\begin{array}{l}\text { (1) Mecanismo de } \\
\text { búsqueda }\end{array}$ & $\begin{array}{l}\text { (2) Mecanismo } \\
\text { de búsqueda, } \\
\text { ocupaciones }\end{array}$ & $\begin{array}{l}\text { (3) Mecanismo de } \\
\text { búsqueda, ocupa- } \\
\text { ciones y regiones }\end{array}$ \\
\hline \multirow{2}{*}{ d_r2 } & & & 0.096 \\
\hline & & & $(0.001)$ \\
\hline \multirow{2}{*}{ d_r3 } & & & 0.047 \\
\hline & & & $(0.001)$ \\
\hline \multirow{2}{*}{ d_r4 } & & & 0.088 \\
\hline & & & $(0.001)$ \\
\hline \multirow{2}{*}{ d_r5 } & & & -0.235 \\
\hline & & & $(0.001)$ \\
\hline \multirow{2}{*}{ d_r6 } & & & 0.000 \\
\hline & & & (omitted) \\
\hline \multirow{2}{*}{ _cons } & 4.036 & 4.840 & 4.799 \\
\hline & (0.004) & $(0.004)$ & $(0.004)$ \\
\hline Adj R-squared & 0.341 & 0.391 & 0.411 \\
\hline $\mathrm{N}$ & 12.701 .906 & 12.701 .906 & 12.701 .906 \\
\hline
\end{tabular}

Nota: todos los coeficientes son significativos al 0.99. Los errores estándar aparecen entre paréntesis. Las variables d_ocup están basadas en los nueve tipos de empleo de sinco, las variables d_r están basadas en la regionalización de Hanson (2003).

Fuente: ENOE (2019).

Los resultados de la regresión por MCO, favorecen la hipótesis de que haber encontrado empleo por medio de amigos y conocidos está correlacionado con una mayor remuneración. La primera estimación describe un incremento del $9.1 \%$ en el salario de los trabajadores a partir de haber encontrado empleo mediante su red de contactos. Sin embargo, no es el medio que mayor prima salarial ofrece. Encontrar trabajo a través de Internet incide un $16.2 \%$ en los ingresos adicionales, seguido de empleo temporal del gobierno (15.2\%) y bolsa privada (13.6\%). La variable servicio público de colocación fue la variable omitida para evitar perfecta multicolinealidad.

En la segunda estimación se incorporan las ocupaciones clasificadas en nueve categorías de acuerdo con la naturaleza, finalidad, funciones, conocimientos y habilidades necesarias para el trabajo, pues estas se asocian con la forma en la cual cada individuo encuentra empleo. ${ }^{7}$ Los nueve grupos de

7 Con base en esta clasificación se pretende captar el efecto diferenciador existente en el mercado laboral entre la oferta de vacantes y su posterior postulación de candidatos. 
acuerdo con el sINco son: (i) funcionarios, directores y jefes; (ii) profesionistas y técnicos; (iii) trabajadores auxiliares en actividades administrativas; (iv) comerciantes, empleados en ventas y agentes de ventas; (v) trabajadores en servicios personales y vigilancia; (vi) trabajadores en actividades agrícolas, ganaderas, forestales, caza y pesca; (vii) trabajadores artesanales; (viii) operadores de maquinaria industrial, ensambladores, choferes y conductores de transporte y, (ix) trabajadores en actividades elementales y de apoyo.

Los resultados corroboran los hallazgos de la primera especificación. Todas las variables se conservan estadísticamente significativas. Internet se vuelve el segundo mecanismo con mayor prima salarial, el primer lugar es ocupado por el empleo temporal del gobierno. En cuanto a la variable central del estudio, la prima salarial a partir de recomendaciones de amigos y conocidos se reduce a $7.5 \%$

En la tercera especificación, se consideran, aparte de los controles por ocupaciones en la ecuación de determinación salarial, un conjunto de regresores referentes a las distintas regiones del país. La pertinencia de controlar por regiones radica en la heterogeneidad espacial de la actividad económica en México, en gran medida, determinada por el grado de exposición al comercio exterior y la cercanía con los Estados Unidos (Hanson, 2003).

La regionalización queda establecida como se muestra a continuación: Frontera: Baja California, Chihuahua, Coahuila, Nuevo León, Sonora, Tamaulipas; Norte: Aguascalientes, Baja California Sur, Durango, Nayarit, San Luis Potosí, Sinaloa, Zacatecas; Centro: Colima, Guanajuato, Hidalgo, Jalisco, Michoacán, Morelos, Puebla, Querétaro, Tlaxcala, Veracruz; Capital: Distrito Federal, México; Sur: Chiapas, Guerrero, Oaxaca; Península: Campeche, Tabasco, Quintana Roo y Yucatán.

Los resultados de la tercera especificación muestran a todas las variables estadísticamente significativas. Internet, junto con el empleo temporal del gobierno, presentan una prima salarial de la misma magnitud (12.4\%), por su parte, amigos y conocidos se reduce a $6.7 \%$, comparándolo con la estimación anterior.

En la tabla 4 se evalúan efectos diferentes del mecanismo de búsqueda de acuerdo a la edad, puesto que el uso de computadoras e Internet es predominante en la población joven (Torres \& Félix, 2017), lo cual tendría implicaciones en la elección del instrumento de búsqueda de empleo. En México, de acuerdo con la Ley del Instituto Mexicano de la Juventud, se

Con estas diferencias nos referimos a los mecanismos mediante los cuales se recluta a operarios, técnicos o artesanos, contrastándolo con los funcionarios y directivos, por mencionar solo algunos. 
considera joven a un individuo cuya edad comprende entre los 12 y 29 años, por lo cual, para efectos de esta investigación los individuos por debajo de los 30 son jóvenes, y aquellos con edades iguales o superiores a este valor, son considerados adultos.

El $60 \%$ de la población mexicana que usa Internet se ubica en un grupo poblacional entre los 6 y los 34 años de edad, de acuerdo con estimaciones propias. En la tabla 4 se aprecia que los años de escolaridad tienen mayor impacto en el salario de las personas más jóvenes. De la misma manera, contar con mayores años de experiencia siendo menores de 30 años, representa un factor relevante para un mejor salario. Ello apuntaría a que entre más jóvenes se integren las personas al mercado laboral, mejores posibilidades tendrán de tener un ingreso elevado a edad temprana. La formalidad en el empleo paga prácticamente igual a los dos grupos de edad. En referencia a la variable de interés, se aprecia que haber encontrado empleo gracias a los contactos, reporta una prima salarial mucho mayor entre las personas mayores de 30 años, $8.6 \%$, comparado con el primer grupo de edad, $0.1 \%$. La comparación por edad destaca al Internet como un medio de búsqueda de empleo crucial para alcanzar salarios elevados entre los jóvenes, representando una prima de $16.4 \%$. Para las personas mayores de 30 años el premio al salario por usar Internet como medio de búsqueda fue de $9.6 \%$.

Tabla 4. Ecuación salarial ajustada en consideración con los mecanismos de búsqueda de empleo diferenciado por edad, México 2019

\begin{tabular}{lcc}
\hline & Menores de 30 años & Mayores de 30 años \\
\hline Años de escolaridad & 0.0522 & 0.0398 \\
& 0.0001 & 0.0001 \\
Sexo & 0.1612 & 0.2611 \\
Estado civil & 0.0006 & 0.0005 \\
& 0.0434 & 0.0417 \\
Experiencia & 0.0006 & 0.0005 \\
Experiencia al cuadrado & 0.0421 & 0.0093 \\
& 0.0002 & 0.0001 \\
Empleo formal & -0.0010 & -0.0001 \\
& 0.0000 & 0.0000 \\
& 0.2032 & 0.2182 \\
\end{tabular}




\begin{tabular}{|c|c|c|}
\hline & Menores de 30 años & Mayores de 30 años \\
\hline \multirow{2}{*}{ Sector agro } & -0.0131 & -0.0093 \\
\hline & 0.0003 & 0.0002 \\
\hline \multirow{2}{*}{ Tamaño de empresa } & -0.0023 & 0.0100 \\
\hline & 0.0002 & 0.0001 \\
\hline \multirow{2}{*}{ Logaritmo de horas trabajadas } & -0.4490 & -0.5072 \\
\hline & 0.0005 & 0.0004 \\
\hline \multirow{2}{*}{ Ir directo al lugar } & -0.0002 & 0.0916 \\
\hline & 0.0068 & 0.0043 \\
\hline \multirow{2}{*}{ bolsa_privada } & -0.0360 & 0.1069 \\
\hline & 0.0087 & 0.0057 \\
\hline \multirow{2}{*}{ Servicios_publicos_colocación } & omitted & omitted \\
\hline & - & - \\
\hline \multirow{2}{*}{ empleo_temporal_gob } & -0.1358 & 0.2615 \\
\hline & 0.0093 & 0.0072 \\
\hline \multirow{2}{*}{ sindicato } & -0.0210 & 0.1013 \\
\hline & 0.0071 & 0.0046 \\
\hline \multirow{2}{*}{ Internet } & 0.1644 & 0.0966 \\
\hline & 0.0070 & 0.0045 \\
\hline \multirow{2}{*}{ medio_comunicion } & -0.0121 & 0.1093 \\
\hline & 0.0068 & 0.0043 \\
\hline \multirow{2}{*}{ Familiar_o_amigo } & 0.0014 & 0.0861 \\
\hline & 0.0068 & 0.0043 \\
\hline \multirow{2}{*}{ Empleo ofrecido } & -0.0299 & 0.0714 \\
\hline & 0.0068 & 0.0043 \\
\hline \multirow{2}{*}{ otro } & -0.0941 & 0.1348 \\
\hline & 0.0076 & 0.0050 \\
\hline Adj R-squared & 0.3643 & 0.4303 \\
\hline $\mathrm{N}$ & 3.831 .207 & 8.870 .699 \\
\hline
\end{tabular}

Nota: todos los coeficientes son significativos al 0.99. Los errores estándar aparecen entre paréntesis. Fuente: INEGI (2019). 
La presente investigación es consistente con trabajos previos. Fontaine (2008) apuntó que la presencia de redes de contactos genera desigualdades de ingreso. Otros trabajos identificaron que el capital social exhibe efectos directos e indirectos sobre los salarios de los inmigrantes al interior de Estados Unidos, para los casos de mexicanos (Aguilera \& Massey, 2003) y mujeres puertorriqueñas (Aguilera, 2005). En lo que respecta al planteamiento de si obtener trabajo a partir de los amigos está asociado con un mejor salario, este estudio identificó que efectivamente es así, y que incluso, el refinamiento de la estimación incrementa ligeramente la significancia estadística de esta variable. Este resultado coincide con lo encontrado en investigaciones previas (Franzen \& Hangartner, 2006).

\subsection{Discusión y limitaciones}

En cierto sentido se podría hablar de que la bolsa privada es el único mecanismo de búsqueda de empleo formal que contribuye positiva y significativamente con el salario de los individuos. Con ello, se refuerza la creencia de que el flujo de información generado por medios informales permite mejores emparejamientos entre personas y firmas.

Encontrar empleo a través de conocidos representa una prima salarial para los individuos. Las personas con contactos tienen mayor acceso a información del mercado laboral (Stigler, 1961, 1962), gracias a ello se enteran de vacantes, condiciones salariales y reciben más ofertas (Addison \& Portugal, 2002). El acceso a información permite a los buscadores de empleo conocer opciones laborales que los medios formales no ofrecen.

Las empresas corren un riesgo al contratar a un empleado nuevo, puede ocurrir que gasten recursos en la capacitación de un recién contratado y luego deban despedirlo y volver a incurrir en costos de selección y capacitación de personal.

Las organizaciones tienen datos sobre las competencias y habilidades de sus trabajadores. A partir de esa información le otorgan un nivel de confianza a cada empleado, si un empleado recomienda a un conocido para una vacante, la empresa toma como referencia el nivel de confianza que tiene su trabajador para aproximar las habilidades que posee la persona recomendada. El proceso permite que los buscadores de empleo sugeridos inicien el proceso de selección de personal con una ventaja sobre aquellos que no fueron recomendados por personas bien valoradas por las empresas.

También puede ocurrir que empleados "mal valorados" por las organizaciones recomienden conocidos para un puesto de trabajo. En este caso, la recomendación jugaría en contra del recomendado y empezaría el proceso de 
selección con una baja valoración, en este tipo de casos, una red de contactos de baja calidad puede resultar perjudicial para los individuos.

El proceso anterior permite que las personas con buenos contactos empaten mejor sus habilidades con los puestos de trabajo disponibles en la empresa en la que tienen un conocido. Sin ese conocido el individuo pudo haber aceptado un empleo con condiciones laborales inferiores. De esta manera, las redes de contactos no solo incrementarían la posibilidad de obtener empleo, sino de conseguir un salario mayor.

Ahora bien, lo anterior es planteado considerando el ingreso a una posición laboral. La persona que entró a trabajar a una organización a partir de un contacto inicia teniendo al menos un conocido. Ello puede facilitar relacionarse con otras personas que le permitan ampliar su red. De esta manera, más gente tiene conocimiento de sus habilidades y competencias. Así, al transcurrir el tiempo, la red ampliada del individuo le puede acercar nuevamente a información sobre vacantes en niveles más elevados en la jerarquía de la empresa. Del mismo modo, quien "sembró" buenas amistades en periodos anteriores puede volver a recurrir a ellas después de un tiempo y obtener mejores perspectivas de empleo y salario.

En referencia a la hipótesis, esta se ve respaldada de manera parcial por los resultados. En lo que respecta a la obtención del empleo a través de las redes de amistad y familiares, los resultados apuntaron a que tal esquema de búsqueda contribuye a un mayor salario. De forma que, la recomendación de amigos o familiares, no solo es un medio popular para obtener un empleo, sino que significa un mayor ingreso para quienes obtienen trabajo a partir de ella. Por otra parte, se observa un mayor ingreso para quienes obtienen empleo mediante Internet, lo cual muestra una ventaja para los individuos habilitados en el uso de esta tecnología, pues desde la búsqueda, implícitamente revelan ser usuarios.

Entre las limitaciones del estudio, se puede señalar que la encuesta utilizada no permite saber con precisión el total de años de experiencia de los encuestados, ni el tiempo que llevan trabajando en su empleo actual. Existen otras variables que pueden incidir en el salario y no son capturadas por la encuesta implementada, tales como dominio del idioma inglés y características de la personalidad (Hill et al., 2016).

\section{Conclusiones}

Con datos de la ENOE 2019, se identificó que encontrar empleo a través de contactos personales o de Internet, representa una prima salarial para los trabajadores. Esto puede estar relacionado con el hecho de que dichos mecanismos 
de búsqueda proveen de mayor información respecto a las vacantes existentes, y en específico al puesto de trabajo, incrementando la posibilidad de que se dé un buen emparejamiento y un mejor salario. Buscar trabajo mediante Internet revela competencias tecnológicas, y con los amigos representa una aproximación de algunas habilidades sociales fundamentales para un buen funcionamiento al interior de la organización.

En el estudio se destaca la relevancia para los menores de 30 años, en cuanto al acceso a un empleo a partir de la búsqueda mediante Internet, mientras que para los que superan los 30 años, existe mayor relevancia por la fortaleza de las redes de amistad.

La red de amigos no solo contribuye con la posibilidad de obtener un empleo, también implica una mayor precepción salarial, esto refleja los resultados positivos de contar con una red amplia de contactos. Lo cual favorece la obtención de información, referencias y recomendaciones en lo que respecta al mercado laboral.

Si bien el uso de Internet como herramienta de búsqueda de empleo es uno de los tres más recurrentes en México, una proporción relativamente baja encuentra empleo por este medio; no obstante, haber hallado trabajo gracias a esta herramienta resultó en un mayor salario para los trabajadores. A partir de los datos presentados en este estudio, tanto empleados como desempleados deberán tomar en consideración la pertinencia de crear y fortalecer redes de contactos adecuados, que les permitan la obtención de un empleo y mejores remuneraciones.

De ese modo, esta investigación contribuye de manera empírica a una mayor comprensión del capital social como un componente clave del mercado de trabajo. En un país como México donde no existe un programa gubernamental de apoyo a desempleados, resulta fundamental identificar las acciones que pueden realizar los buscadores de empleo para alcanzar mejores salarios. Otro aporte radica en que la investigación permite vislumbrar mejor las dinámicas del mercado de trabajo de años recientes, donde las reconfiguraciones de los métodos de búsqueda de empleo impactan a las diferentes generaciones, ya que se mostraron efectos diferentes para jóvenes, adultos y adultos mayores. Estudios futuros pueden profundizar el análisis integrando más variables relacionadas con habilidades tecnológicas, sociales y de personalidad.

\section{Referencias}

Addison, J. T., \& Portugal, P. (2002). Job search methods and outcomes. Oxford Economic Papers, 54(3), 505-533. https://doi.org/10.1093/oep/54.3.505 
Aguilera, M. B. (2002). The impact of social capital on labor force participation: Evidence from the 2000 Social Capital Benchmark Survey. Social Science Quarterly, 83(3), 853-874. https://doi.org/10.1111/1540-6237.00118 Aguilera, M. B. (2005). The Impact of Social Capital on the Earnings of Puerto Rican Migrants. Sociological Quarterly, 46(4), 569-592. https://doi. org/10.1111/j.1533-8525.2005.00027.x

Aguilera, M. B., \& Massey, D. S. (2003). Social Capital and the Wages of Mexican Migrants: New Hypotheses and Tests. Social Forces, 82(2), 671-701. https://doi.org/10.1353/sof.2004.0001

Bachmann, R., \& Baumgarten, D. (2012). How Do the Unemployed Search for a Job? Evidence from the Eu Labour Force Survey. IZA Discussion Paper (6753), 1-28. https://doi.org/10.1186/2193-9012-2-22

Barceinas Paredes, F. (2002). Rendimientos privados y sociales de la educación en México. Economía Mexicana Nueva Época, XI, (2), 333-390. http:// hdl.handle.net/11651/1760

Bowlus, A. J., \& Ontario, W. (1997). A Search Interpretation of Male- Female Wage Differentials. Journal of Labor Economics, 15(4), 625-657. https:// doi.org/10.1086/209840

Bracho, T., \& Zamudio Carrillo, A. (1994). Los rendimientos económicos de la escolaridad en México, 1989. Economía Mexicana Nueva Época, 3(2), 345-377. http://hdl.handle.net/11651/4240

Bridges, W. P., \& Villemez, W. J. (1986). Informal Hiring and Income in the Labor Market. American Sociological Review, 51(4), 574-582. https://doi. org $/ 10.2307 / 2095589$

Burgos, B., \& Mungaray, A. (2007). Apertura externa, inequidad salarial y calificación laboral en México, 1984-2002. Problemas del Desarrollo, 39(152), 87-111. https://doi.org/10.22201/iiec.20078951e.2008.152.7705

Calvó-Armegol, A., \& Jackson, M. O. (2004). The Effect of Social Networks on Employment and Inequality. The American Economic Review, 94(3), 426-454. https://doi.org/10.4057/jsr.60.279

Charles-Leija, H., Torres, A., \& Castro, D. (2018). Efectos del Capital Social en el empleo en México. Revista de Economía Institucional, 20(38), 263-283. https://doi.org/10.18601/01245996.v20n38.11

Dale, S. B., \& Krueger, A. B. (2002). Estimating the Payoff to Attending a More Selective College: An Application of Selection on Observables and Unobservables. The Quarterly Journal of Economics, 117(4), 1491-1527. https://doi.org/10.1162/003355302320935089

Empleo. (2020). Programa de Empleo Temporal. Portal de empleo. https:// www.gob.mx/bienestar/acciones-y-programas/programa-de-empleotemporal 
Esquinca, M. T., \& Valdivia, J. M. (2006). La afiliación sindical y premio salarial en México. En E. de la Garza \& C. Salas Pérez (Eds.), La situación del trabajo en México (pp. 459-485). Plaza y Valdés.

Fontaine, F. (2008). Why are similar workers paid differently? the role of social networks. Journal of Economic Dynamics and Control, 32(12), 39603977. https://doi.org/10.1016/j.jedc.2008.04.009

Franzen, A., \& Hangartner, D. (2006). Social networks and labour market outcomes: The non-monetary benefits of social capital. European Sociological Review, 22(4), 353-368. https://doi.org/10.1093/esr/jcl001

Goyal, S. (2007). Connections: an introduction to the economics of networks. Princeton University Press.

Hanson, G. H. (2003). What has happened to wages in Mexico since NAFTA? (Working Paper n. ${ }^{\circ}$ 9563). National Bureau of Economic Research. https:// doi.org/10.3386/w9563

Hellerstein, J. K., Kutzbach, M. J., \& Neumark, D. (2013). Do labor market networks have an important spatial dimension? (Working Paper $\mathrm{n} .{ }^{\circ}$ 18763). National Bureau of Economic Research. https://doi.org/10.1016/j. jue.2013.03.001

Hill, P. L., Turiano, N. A., Mroczek, D. K., \& Burrow, A. L. (2016). The value of a purposeful life: Sense of purpose predicts greater income and net worth. Journal of Research in Personality, 65, 38-42. https://doi.org/10.1016/j. jrp.2016.07.003

Huesca, L., \& Ochoa, G. (2016). Desigualdad salarial y cambio tecnológico en la Frontera Norte de México. Problemas Del Desarrollo, 47(187), 165-188. https://doi.org/10.1016/j.rpd.2016.10.006

Instituto Nacional de Estadística y Geografía (INEGI). (2019). Encuesta Nacional de Ocupación y Empleo (ENOE), trimestre III. https://www.inegi.org.mx/ programas/enoe/15ymas/\#Tabulados

Kuhn, P., \& Mansour, H. (2014). Is Internet Job Search Still Ineffective?. The Economic Journal, 124(581), 1213-1233. https://doi.org/10.1111/ecoj.12119

Loury, L. D. (2006). Some Contacts are More Equal than Others: Informal Networks, Job Tenure, and Wages. Journal of Labor Economics, 24(2), 299-318. https://doi.org/10.1086/499974

Marmaros, D., \& Sacerdote, B. (2002). Peer and social networks in job search. European Economic Review, 46(4-5), 870-879. https://doi.org/10.1016/ S0014-2921(01)00221-5

Méndez, A., Castro Lugo, D., \& Mena, E. (2018). Efectividad de los mecanismos de búsqueda de empleo en el mercado laboral mexicano. Ensayos de Economía, 28(52), 77-100. https://doi.org/10.15446/ede.v28n52.72369 
Mincer, J. (1974). Schooling, experience, and earnings (Working Paper n. ${ }^{2}$ ). National Bureau of Economic Research.

Montgomery, J. D. (1991). Social Networks and Labor-Market Outcomes: Toward an Economic Analysis. The American Economic Review, 81(5), 1408-1418. https://doi.org/10.1016/j.jcorpfin.2007.04.005

Pellizzari, M. (2010). Do friends and relatives really help in getting a good job? Industrial and Labor Relations Review, 63(3), 494-510. https://doi. org/10.1177/001979391006300307

Rath, T., \& Harter, J. (2010). The Economics of Wellbeing. Gallup Report. https:// fbo1.typepad.com/salt_lake_city_ut_6181162/SLC_files/PDFs/The_Economics_of_Wellbeing.pdf

Rees, A. (1966). Information Networks in Labor Markets. The American Economic Review, 56(1), 559-566. https://www.jstor.org/stable/1821319

Rodríguez-Villalobos, M., \& Rangel-González, E. (2020). Social networks and job quality in Mexico: 2005-2019. Heliyon, 6(6). https://doi.org/10.1016/j. heliyon.2020.e04127

Rosenbaum, J. E., Deluca, S., Miller, S. R., \& Roy, K. (1999). Pathways into Work: Short- and Long-term Effects of Personal and Institutional Ties. Sociology of Education, 72(3), 179-196. https://doi.org/10.2307/2673228

Stigler, G. J. (1961). The Economics of Information. The Journal of Political Economy, 69(3), 213-225. https://doi.org/10.1086/258464

Stigler, G. J. (1962). Information in the Labor Market. The Journal of Political Economy, 70(5-2), 94-105. https://doi.org/10.1086/258727

Torres, A., \& Félix, G. (2017). El uso de Internet y su relación con los salarios en México: un análisis no paramétrico. En Los retos del cambio económico actual: revisión y aplicaciones para el caso mexicano (pp. 31-50). Universidad Autónoma de Chihuahua; Ediciones de Laurel.

Villarreal, E. M. (2010). Evolución histórica de los rendimientos educativos en México: 1987-2009. Investigaciones de Economía de la Educación, 5(5), 223244. https://repec.economicsofeducation.com/2010zaragoza/05-11.pdf 